\title{
Mechanics of Twisted DNA Molecule Adsorbed on a Biological Membrane
}

\author{
Radouane El Kinani' ${ }^{1}$ Hamid Kaidi², Noureddine Barka1 \\ ${ }^{1}$ Departement of Mathematics, Computer Science and Engineering, University of Quebec at Rimouski, \\ Rimouski, Canada \\ ${ }^{2}$ Laboratory of Scientific Research and Pedagogical Development, CRMEF, Meknes, Morocco \\ Email: RadouaneElkinani@uqar.ca, hamidkaidi@gmail.com
}

How to cite this paper: El Kinani, R., Kaidi, H. and Barka, N. (2020) Mechanics of Twisted DNA Molecule Adsorbed on a Biological Membrane. Open Journal of Biophysics, 10 , 129-149.

https://doi.org/10.4236/ojbiphy.2020.103011

Received: May 10, 2020

Accepted: July 5, 2020

Published: July 8, 2020

Copyright $\odot 2020$ by author(s) and Scientific Research Publishing Inc. This work is licensed under the Creative Commons Attribution International License (CC BY 4.0).

http://creativecommons.org/licenses/by/4.0/

\section{(c) (i) Open Access}

\begin{abstract}
DNA is the carrier of all cellular genetic information and increasingly used in nanotechnology. The study of DNA molecule achieved in vitro while submitting the DNA to all chemicals agent capabilities to destabilize links hydrogen, such as $\mathrm{pH}$, temperature. In fact, the DNA enveloped in the membrane cellular, so it is legitimate to study the influence of membrane undulations. In this work, we try to show that the fluctuations of the membrane can be considerate as a physics agent is also capable to destabilize links hydrogen. In this investigation, we assume that each pair base formed an angle $\alpha_{n}$ with the membrane's surface. We have proposed a theoretical model, and we have established a relationship between the angle formed by the pair base $\theta_{e q}$ and angle $\alpha_{n}$ formed by the membrane and each pair base. We assume that DNA and biomembrane interact via a realistic potential of Morse type. To this end, use is made of a generalized model that extends that introduced by M. Peyrard and A. R. Bishop in the past modified by M. Zoli. This generalized model is based on the resolution of a Schrödinger-like equation. The exact resolution gives the expression of the ground state, and the associated eigenvalue (energy) that equals the free energy, in the thermodynamic limit. First, we compute the denaturation temperature of DNA strands critical temperature. Second, we deduce all critical properties that mainly depend on the parameters of the model, and we quantify the effects of the membrane undulations. These undulations renormalize all physical quantities, such as harmonic stacking, melting temperature, eigenfunctions, eigenvalues and regular part of specific heat.
\end{abstract}

\section{Keywords}

Biomembrane, DNA Molecule, Denaturation, Interactions, Critical Properties 


\section{Introduction}

The Deoxyribose-Nucleic-Acid molecule (DNA) is considered among the most important biological systems for living beings. Since Watson and Crick unveiled its topological structure [1]. This molecule has become a vast field of research. All the genetic information that controls the cell reproduction is coded there, constituting the most sensitive molecule of the cellular nucleic.

A chromosome with protein components is a complete DNA molecule. Amino acids are assembling in the right order to produce the protein. This assembly is triggered by a chemical message carried by the DNA of each gene, tells the cell how to organize this assembly. Monomers that constitute the molecule of DNA are called nucleotides contained information. The structure of the DNA molecule consists of a base and a skeleton alternating phosphate ions and sugar molecules. In DNA, there are four different nucleotides, namely guanine (G), adenine (A), cytosine (C) and thymine (T), whose repeated stacks are formed by AT or GC. The stability of the double helix, i.e. its denaturation resistance depends on its sequence: the G-C rich sequences have a greater resistance to denaturation than the A-T rich one. Because the opening of the G-C base requires the breaking of three hydrogen bonds, while the opening of a pair of A-T involves only two hydrogen bridges. In the case of thermal denaturation, the mean denaturation temperature of a given chain is effectively related to the percentage of G-C contained in the sequence [2].

Several works have been interesting by the study of DNA-interface interactions considered as a subject of general nature. DNA interactions with surfaces can be repulsive leading to confinement or depletion [3] [4]. In this situation, there exist a repulsive interaction between negatively charged phosphates of DNA and phospholipids of membranes and contributes to enclose the genetic material within the cell [5]. The depletion forces also confine DNA within viral capsids [6]. To indicate the repulsive interactions are a common requirement in micro and nano-fluidics [7]. On the one hand, the attractive interactions lead to adsorption on a surface. The adsorption studied is done by $\mathrm{x}$-ray reflectivity [8]. In some recent work [9], a theoretical analysis of such scenarios is based on the self-consistent field theory approach. In this work, they treated the importance of the charge correlation effect.

The thermodynamic magnitudes of DNA are affected by several factors, for example the thermodynamic properties with the interaction of the solvent on the nonlinear dynamical structure of a DNA segment [10], by using a time-independent perturbation approach. Also, for a short fragment of heterogeneous DNA with a stabilizing solvent interaction term, the technique used in this case is the imaginary time path integral formalism that is applied to a nonlinear Hamiltonian [11]. These nonlinearities in DNA dynamics were first emphasized by Englander et al. [12] who interpreted the formation of temporary open segments of base pairs as moving defects propagating coherently along the backbone of the molecule. This phenomenon has been treated like a bubble dynamics in a random DNA sequence, in a study of the localization of denaturation [13]. 
The denaturation transition is done by the hydrogen bond rupture between two pair bases, so the set of the two strands is transformed into a single strand. This phenomenon is done by several factors, for example, when a sufficiently heated solution of DNA. The temperature at which the DNA strands are half denatured, meaning half double-stranded, half single-stranded, is called the melting temperature $\left(T_{m}\right)$ or denaturation temperature $\left(T_{d}\right)$. The amount of strand separation, or melting, is measured by the absorbance of the DNA solution at $260 \mathrm{~nm}$ (absorb in the ultraviolet). Nucleic acids absorb light at this wavelength because of the electronic structure in their bases, but when two strands of DNA come together, the proximity of the bases in the two strands quenches some of this absorbance. As the DNA becomes denatured, its absorption of light ultraviolet increases, when the two strands separate, this quenching disappears, and the absorbance rises $30 \%-40 \%$. This is called Hyperchromicity. The Hypochromic effect is the effect of stacked bases in a double helix absorbing less ultra-violet light.

For a quantitative study, Thierry Dauxois and Michel Peyrard presented a model for the dynamical structure of DNA that can be considered as an extension of the usual Ising-like statistical approach to the melting curves. Also, Thierry Dauxois and Michel showed by numerical simulation method at constrained temperature show that it provides a good qualitative description of the collective motions of the base pairs, including their large-amplitude fluctuational openings and the emergence of the denaturation bubbles from the thermal fluctuation [14].

In this paper, we focus on the statistical of a DNA molecule on the fluid membrane, our aim is to determine the quantity physics. Our system physics constituted by a fluid membrane fluctuating around a horizontal, plane on this the latter adsorbed a DNA molecule. Firstly, we determine the partition function, from which we establish the free energy of the system. Based on the latter, we derive the magnitudes thermodynamics such as heat energy. As results, we have showed that the constant of harmonic stacking is renormalized, and the DNA becomes more elastic. This constant depends crucially on the membrane parameters. The thermal fluctuations of membrane modify the depth of interaction potential of staking and inverse length. On the other hand, we concluded that the fluctuations of the membrane increase the denaturation temperature. In the transition point we constant that the average separation between base-pair and specific heat are diverging.

The remaining of the presentation proceeds as follows. In Section 2, we presented the physical system with its parameters, and the Section 3 is reserved to formalism for partition function and free energy, the exact study of the denaturation transition is presented in the Section 4, Some concluding remarks are drawn in Section 5.

\section{Description of the Physical System and the Basic Equation}

The denaturation transition can be achieved in vitro, while submitting the DNA 
to all chemicals or physical agent capabilities to destabilize links hydrogen, such as $\mathrm{pH}$, temperature, certain solvents, alkaline agents, high ionic concentrations [10], in this paper, the authors investigated the effect of solution concentrations on physical quantities such as specific heat, entropy, melting temperature, and the mean hydrogen bonding stretching.

In the living cells, the DNA molecule is compacted in a biological membrane. Quantitative understanding and optimization of its functions require precise experimental characterization and accurate modelling of DNA properties on the biological membrane. The legitimate question, how the thermal fluctuations of the membrane affect the denaturation temperature of the DNA molecule, and its influence on thermodynamics quantities. Due to the nature of electrically charged DNA, as well as superficially charged cell membrane leads to the phenomenon of adsorption [9]. Therefore, in this investigation, we assume a DNA-molecule adsorbed on a fluctuating membrane, such as each base pair of index $n$, formed an angle $\alpha_{n}$, with the plan of the membrane (see Figure 1). In this model, the interaction between DNA-molecule and membrane per area has an attraction, as well as hard wall repulsion for a base pair residing at a point on the membrane surface.

On the other hand, we introduce the twist angle $\theta_{e q}=c t e$, for the pairs bases adsorbed, we take the equilibrium twist angle value, in our situation $\theta_{e q}=\pi / 10$, we choose the model of Dauxois-Peyrard-Bishop (DPB) modified by Zoli [11]. This author introduced the rotation angle between adjacent bases $n$, and $n-1$ along the DNA backbone. The twist angle between adjacent base $n$, and $n-1$, we assume constant, therefore

$$
\left\{\begin{array}{l}
\theta_{e q}=\theta_{n}-\theta_{n-1} \\
\theta_{e q}=\theta_{n+1}-\theta_{n}
\end{array}\right.
$$

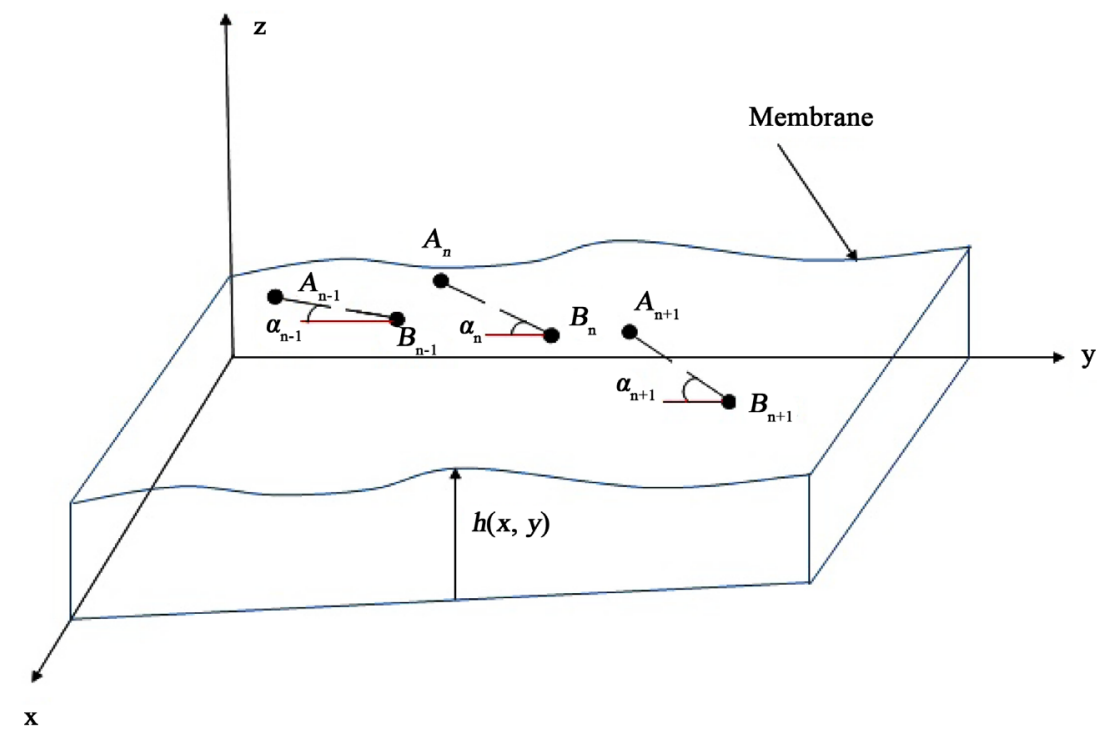

Figure 1. Biological membrane with a twisted DNA adsorbed. 
We consider the adsorption of DNA on the membrane as the adsorption of copolymer on the substrate, which forms loops, so there are a few base-pair are adsorbing on this membrane for each loop. In the other hand, some work show that [15] the distance between adjacent bases in closed case of base pair $(\sim 3.3 \AA)$ is smaller than the distance of separation between base pairs $(\sim 18 \AA)$, but it's clear that length between base pair in the opening state is greater than the distance between two base, so with these considerations we assume that the two bases adjacent are coplanar. We take for example the first adjacent bases (base 1 and 2), we can establish the first relation between the angels $\alpha_{1}$ and $\alpha_{2}$, such as $\alpha_{2}=\theta_{e q}+\alpha_{1}$ and the second adjacent bases (base 2 and 3 ), we find $\alpha_{3}=2 \theta_{e q}+\alpha_{1}$, etc. (see Figure 2), finally, we get the relationship between $\theta_{e q}$ and $\alpha_{n}$

$$
\alpha_{n}=(n-1) \theta_{e q}+\alpha_{1}
$$

where $\alpha_{1}$ is the first angle between adsorbed base pair and membrane, from this relationship, we can determine the maximum number of base pairs (bp) adsorbed on the membrane and contribute to the denaturation transition phenomena by the following relation:

$$
n_{\max }=\frac{1}{\theta_{e q}}\left(\alpha_{n}-\alpha_{1}\right)
$$

As long as adsorption takes place with a few monomers (we take for example $\left.n_{\max }=5\right)$ to form the brush, we can choose for example $\alpha_{1}=\pi / 10$, and the number of base pair which contribute to adsorption is $0<n \leq 5$, we deduct that $0 \leq \alpha_{n} \leq \pi / 2$. In the absence of the membrane, the number of base pairs that contribute to the denaturation transition is of the order of $20 \mathrm{bp}$ for $N \sim 10^{3}$ total number of base pairs [13].

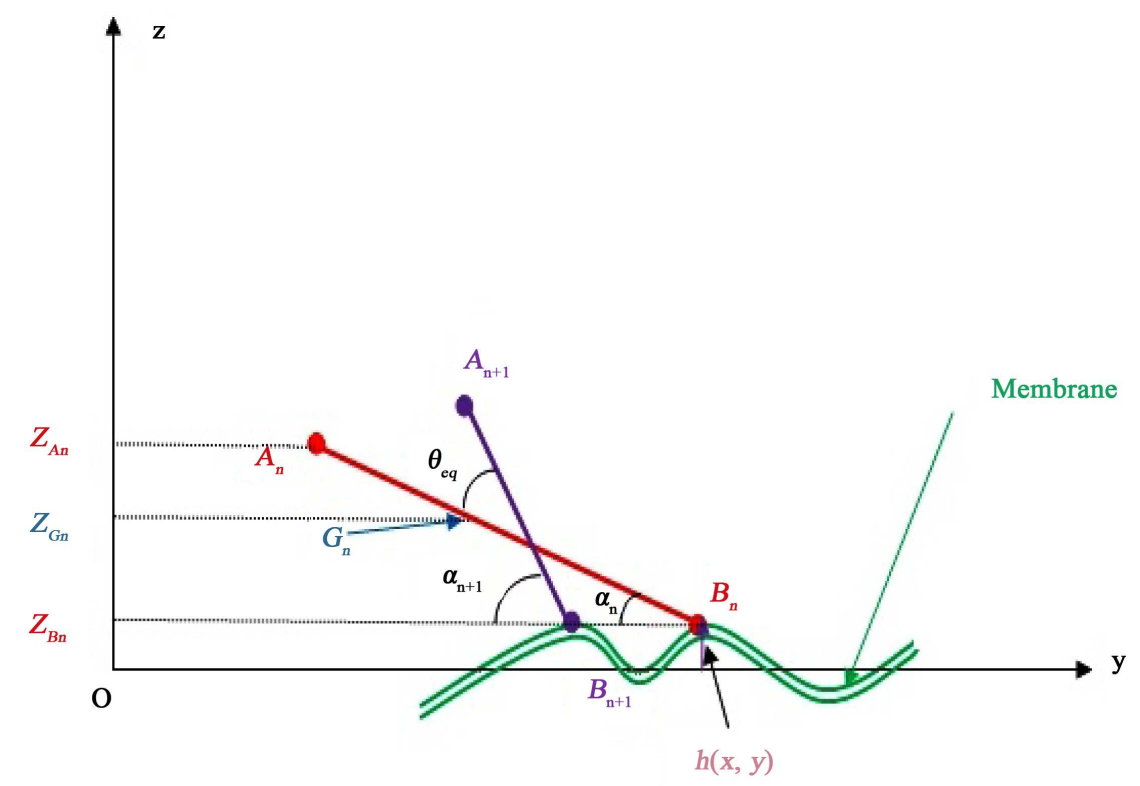

Figure 2. Cross-section of a membrane with an adsorbed DNA molecule. 
The double-strand is denoted by the base sequence $A_{1} B_{1} \cdots A_{n} B_{n}$ (where $A_{k}$ and $B_{k}$ are the nucleotides with $A_{k}, B_{k} \in\{\mathrm{A}, \mathrm{C}, \mathrm{G}, \mathrm{T}\}$, of one of the strands, ordered from the 5 'to 3 'end. The distance between the mass center of the base pairs and a point situated on the surface of the membrane is given by barycenter technique $z_{G n}=z_{A n}+z_{n} m \sin \alpha_{n}$, where $m=m_{B n} /\left(m_{A n}+m_{B n}\right)$ is the reduced mass, $z_{A n}$ is the abscissa of the nucleotide $A_{n}$, and $z_{n}$ is the distance between two nucleotides $A_{n}$ and $B_{n}$ (i.e. is the length of the hydrogen bond), since we are interested in the bases of pairs, that is adsorbed on the membrane, we take $z_{A n}$ or $z_{B n}$ equal the fluctuation amplitude of the membrane $h(x, y)$

\subsection{The Hamiltonian of Membrane}

The membrane is a flexible, continuous surface that has a size $L$ and area $\mathcal{S}=L^{2}$. Membrane local position vectors are, in the Monge representation, $\boldsymbol{r}=x \hat{\boldsymbol{x}}+y \hat{\boldsymbol{y}}+h(x, y) \hat{\boldsymbol{z}}$, where $h(x, y)$ is the height of the membrane. The position of the (almost flat) membrane is specified through the displacement field $h(x, y)$. The surface fluctuates around the horizontal plane $z=0$. The equilibrium statistical mechanic of the membrane is based on the Canham-Helfrich Hamiltonian [16] [17]

$$
H_{m}=\frac{1}{2} \int_{S} \mathrm{~d}^{2} \rho\left[\kappa\left(\nabla^{2} \rho h(\rho)\right)^{2}+\sigma(\nabla \rho h(\rho))^{2}\right]
$$

where $\kappa\left(\simeq 20-50 k_{B} T\right)$, is the membrane bending rigidity, $\sigma$ is the microscopic membrane surface tension, and $\rho=x \hat{x}+y \hat{y}$ is the projection of $r$ in the reference plane, $\nabla_{\rho}=\left(\partial_{x}, \partial_{y}\right)$ is the cartesian operator in the basic $(x, y)$, and $\mathcal{S}$ is the projected membrane area. Although we mainly refer to membranes, our development below with this Hamiltonian applies to a variety of membranes and interface including fluid membranes $(\sigma \simeq 0)$ and elastic interface $(\kappa \simeq 0)$.

\subsection{Hamiltonian Model of DNA}

In our investigation, we choose the model (DPB) modified by Zoli [11] [14], thus generalizing the DPB Hamiltonian, is given by

$$
\mathcal{H}_{D N A-z}=\sum_{n=1}^{N}\left[\frac{m \dot{z}_{n}^{2}}{2}+\frac{K}{2}\left(z_{n}^{2}-2 z_{n} z_{n-1} \cos \theta_{e q}+z_{n-1}^{2}\right)+D_{0}\left(\mathrm{e}^{-a z_{n}}-1\right)^{2}\right],
$$

where $m$ is the base pair reduced mass, $\dot{z}_{n}=\mathrm{d} z_{n} / \mathrm{d} t$ is the nucleotide velocity, $K$ is the harmonic stacking, $D_{0}$ and $a$ are the pair dissociation energy and the inverse length setting the hydrogen bond potential range for the $n$-th base pair. The first term is the kinetic energy, the second term is the potential energy of the longitudinal links and the last term represents the dissociation energy of the pair (for a very deep reading see the Ref. [14]). The settings in this Hamiltonian are adjusted to match our problem $\left(K=4 \mathrm{eV} \cdot \mathrm{nm}^{-2}, D_{0}=0.15 \mathrm{eV}, a=0.63 \mathrm{~nm}^{-1}\right.$, [18]).

\subsection{Interaction between DNA Molecule and Membrane}

The DNA molecule and membrane are subject to mutual interaction which we 
denote by

$$
V_{D N A-m}=\sum_{n=1}^{N} \int \mathrm{d}^{2} \rho U\left(z_{G n}-h(\rho)\right)
$$

the sum of all the base pairs, and continued sum covers the surface of the membrane. The potential that can be used to model interactions such as the interaction between an atom and a surface is the Morse potential. Because of its simplicity, it is not used in modern spectroscopy [19]. However, its mathematical form has inspired the MLR (Morse/Long Range) potential, which is the most used potential energy function for spectroscopic data fitting [19]. The potential chose to mimic the adsorption potential by a simpler one, the Morse potential

$$
U\left(z_{G n}-h(\rho)\right)=\frac{U_{0}}{\mathcal{S}}\left[1-\exp \left(-b_{0}\left(z_{G n}-h(\rho)\right)\right)\right]^{2},
$$

we replace $z_{G n}$ by its expression in this potential we find

$$
U\left(z_{n}\right)=\frac{U_{0}}{\mathcal{S}}\left[1-\exp \left(-b z_{n} \sin \alpha_{n}\right)\right]^{2},
$$

with $b=m b_{0}$ and $\mathcal{S}$ is the membrane's surface. In our model, the interaction $U\left(z_{n}\right)$ between base pair and membrane per area has an attraction, as well as hard wall repulsion (impenetrability) for a base pair residing at a position $(\rho, h(\rho))$ on the surface. Such impenetrability by DNA molecule is conceivable even in fluid membrane unless the striking barrier formed by the lipid bilayer self-assembly is disturbed by a strong attraction, (i.e. between hydrophobic segment in the DNA-chain and inner hydrophobic part of the bilayer) leading to chain penetration into the membrane, the choice of the Morse potential to ensure the adsorption phenomena, in a similar work [20], the potential chosen is the hard square-well with depth $U_{0}$ and width $b_{0}$. The Parameters for the Morse potential are adjusted to have a good match at the minimum with the true potential $\left(b_{0}=0.3 \mathrm{~nm}^{-1}, U_{0}=0.05 \mathrm{eV}\right)$.

\section{The Partition Function of System and Free Energy}

For a chain containing $N$ units (nucleotide), the classical partition function of the system may be factored as

$$
\begin{aligned}
\mathcal{Z} & =\int \mathcal{D} p_{n} \mathcal{D} h(\rho) \mathcal{D} z_{n} \mathcal{D} \alpha_{n} \exp \left[-\beta\left(\mathcal{H}_{m}+\mathcal{H}_{D N A}+V_{D N A-m}\right)\right] \\
& =\mathcal{Z}_{p} \times \int \mathcal{D} h(\rho) \exp \left[-\beta\left(\mathcal{H}_{m}\right)\right] \times \int \mathcal{D} z_{n}\left\{\mathrm{e}^{-\beta \mathcal{H}_{D N A}} \int \mathcal{D} \alpha_{n} \exp \left[-\beta\left(V_{D N A-m}\right)\right]\right\}
\end{aligned}
$$

where we define as usual $\beta=1 / k_{B} T$, with $T$ the absolute temperature, $k_{B}$ Boltzmann's constant. The momentums parts are readily integrated to give the familiar kinetic factor for $N$ particles $\mathcal{Z}_{P}=\left(2 \pi m k_{B} T\right)^{N / 2}$. The sum on the variable $\alpha_{n}$ in Equation (6) is independent of the other variables, so we can compute this sum separately, therefore we get the following relation (see Appendix) [21]

$$
\begin{aligned}
& \int_{0}^{\pi / 2} \mathcal{D} \alpha_{n} \mathrm{e}^{\left[-\beta U_{0}\left(1-\exp \left(-b z_{n} \sin \alpha_{n}\right)\right)^{2}\right]} \\
& =\frac{1}{b z_{n}}\left[\frac{1}{\sqrt{\beta U_{0}}} \mathrm{e}^{-\beta U_{0}\left(1-\mathrm{e}^{-b z_{n}}\right)^{2}}\right] \times \sum_{k=0}^{\infty} \frac{2^{k}\left[\sqrt{\beta U_{0}}\left(1-\mathrm{e}^{-b z_{n}}\right)\right]^{2 k+1}}{(2 k+1) !}
\end{aligned}
$$


we are restricted to the first order, and, we have been interested in the phenomenon of breathing, which is affected near of point of equilibrium, then $z_{n}$ is weak. Thus, we get the potential expressed by the following relationship

$$
\begin{aligned}
& \int_{0}^{\pi / 2} \mathcal{D} \alpha_{n} \exp \left[-\beta U_{0}\left(1-\exp \left(-b z_{n} \sin \alpha_{n}\right)\right)^{2}\right] \\
& \simeq \exp \left[-\beta U_{0}\left(1-\mathrm{e}^{-b z_{n}}\right)^{2}\right]
\end{aligned}
$$

The partition function (6) becomes

$$
\begin{aligned}
\mathcal{Z}= & \left(2 \pi m k_{B} T\right)^{N / 2} \mathcal{Z}_{0} \int \mathcal{D} z_{n} \exp \left\{-\beta \sum_{n=1}^{N}\left[\frac{K}{2}\left(z_{n}^{2}-2 z_{n} z_{n-1} \cos \theta_{e q}+z_{n-1}^{2}\right)\right]\right\} \\
& \times \exp \left\{-\beta\left[+D_{0}\left(1-\mathrm{e}^{-a z_{n}}\right)^{2}+U_{0}\left(1-\mathrm{e}^{-b z_{n}}\right)^{2}\right]\right\} \\
= & \left(2 \pi m k_{B} T\right)^{N / 2} \mathcal{Z}_{0} \times \mathcal{Z}_{z},
\end{aligned}
$$

where $\mathcal{Z}_{0}=\int D h(\rho) \exp \left(-\beta \mathcal{H}_{m}\right)$ is the partition function of the free membrane for developing the part related to the twist angle in the last equation, we are introducing sum and difference coordinates $d_{n}=\left(z_{n}+z_{n-1}\right) / 2$ and $\delta_{n}=z_{n}-z_{n-1}$. In this work, we also assume the twist angle is very low, which affects the harmonic part, becomes $-\beta K\left(z_{n}-z_{n-1}\right)^{2} / 2-\beta K \theta_{e q} z_{n} z_{n-1} / 2$. Therefore $\mathcal{Z}_{n}$ can be expressed in the form

$$
\mathcal{Z}_{z}=\int_{-\infty}^{+\infty} \sum_{n=1}^{N} \mathrm{~d} z_{n} \mathrm{e}^{-\beta E_{p o t}\left(z_{n}, z_{n-1}\right)},
$$

where $E_{p o t}$ denotes the total potential energy in $z_{n}$. This integral can be evaluated exactly in the thermodynamic limit of a large system $(N \rightarrow \infty)$ using the eigenfunctions and eigenvalues of the transfer-integral operator [22] [23] [24]

$$
\int \mathrm{d} z^{\prime} \mathrm{e}^{-\beta E_{p o t}\left(z, z^{\prime}\right)} \varphi_{i}\left(z^{\prime}\right)=\mathrm{e}^{-\beta \epsilon_{i}} \varphi_{i}\left(z^{\prime}\right) .
$$

To solve this integral we change the variable of integration from $z$ to $Z$ (where $\left.z^{\prime}=d+\delta\right)$, in what follows, we assume $d_{n} \approx z$, and introducing $\varphi_{i}(d+\delta) \approx \varphi_{i}(z)+\varphi_{i}^{\prime}(z) \delta+\varphi_{i}^{\prime \prime}(z) \delta^{2} / 2$, and performing the Gaussian integrals over $\delta$. From this equation, with the new parameter, in the continuum limit approximation, the transfer integral eigenvalue problem can be reduced the following Schrödinger-like equation

$$
\frac{\mathrm{d}^{2} \varphi_{i}}{\mathrm{~d} z^{2}}+2 \beta^{2} K^{\prime}\left(\epsilon_{i}-s-V_{t o t}(z)\right) \varphi_{i}(z)=0,
$$

where the harmonic stacking $K^{\prime}$ depend on the $\theta_{e q}$ defined by $K^{\prime}=K\left(1-\theta_{e q}^{2} / 4\right), \quad V_{t o t}(z)=\left(K \theta_{e q}^{2} / 2\right) z^{2}+D_{0}\left(\mathrm{e}^{-a z}-1\right)^{2}+U_{0}\left(1-\mathrm{e}^{-b z}\right)^{2} \quad$ is the new potential and $s$ is the modified entropy by the presence the angle twist

$$
s=s_{0}+\frac{1}{2 \beta} \ln \left(1-\frac{\theta_{e q}^{2}}{4}\right),
$$

with $s_{0}=(1 / 2 \beta) \ln (\beta K / 2 \pi)$ is the entropy determined by $T$. Dauxois et al. [14], from this relation we can deduce that twist angle minimizes the entropy of 
the DNA-molecule and the harmonic stacking. The first term is harmonic potential, we can be transformed as a Morse potential at high temperature, we find potential with six pertinent parameters $\left(a, b, c, D_{0}, D_{0}^{\prime}, U_{0}\right)$ given by the following relation

$$
V_{t o t}(z)=D_{0}\left(1-\mathrm{e}^{-a z}\right)^{2}+D_{0}^{\prime}\left(1-\mathrm{e}^{-c z}\right)^{2}+U_{0}\left(1-\mathrm{e}^{-b z}\right)^{2},
$$

where $D_{0}^{\prime}=\theta_{e q}^{2} / 2$ is the depth also, and $c=\sqrt{K \beta}$, this parameter is homogeneous to the inverse of a length. For a temperature $T=320 \mathrm{~K}$, we can estimate the values of the depth, and the range respectively, $D_{0}^{\prime} \simeq 5.03 \times 10^{-3} \mathrm{eV}$ and $c \simeq 0.12 \mathrm{~nm}^{-1}$. This potential is plotted in Figure 3 , this potential reflects standard expression for chemical bonds and, moreover, it has the appropriate qualitative shape: 1) it includes a strong repulsive part for $z<0$ corresponding to the steric hindrance 2) it has a minimum at the equilibrium position $z=0,3$ ) it becomes flat for large $z$, giving a force between the bases that tends to vanish, as expected when the bases are very far apart, this feature allows a complete dissociation of the base pair, which would be forbidden if we had chosen a simple harmonic potential.

The shape of this potential is more adapted to our problem because it ensures the phenomenon of breathing of the DNA-molecule near its minimum has a harmonic form. So, this potential can be well fitted to the Morse potential, we can be rewritten as follows

$$
V_{\text {tot }}(z)=D(1-\exp (-\alpha z))^{2} .
$$

Using the relation (13), to find the zero and minimum of this potential $\left(V_{t o t}\left(z_{0}\right)=0\right)$, then we can find the expression of the depth and the minimum $\left(\mathrm{d} V_{\text {tot }}\left(z_{m}\right) / \mathrm{d} z=0\right)$. All the parameters depend on the six pertinent parameters, we find

$$
D=D_{0}+D_{0}^{\prime}+U_{0},
$$

is the depth of the Morse potential, and $\alpha$ defines the range parameter given by

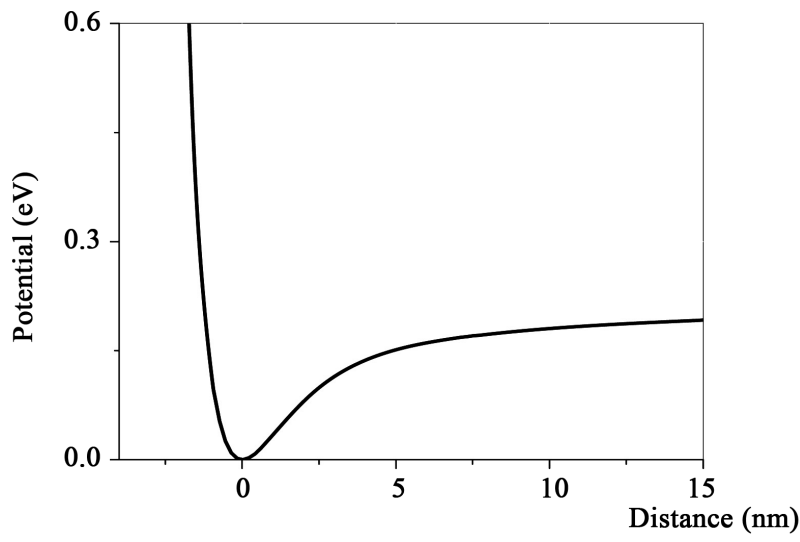

Figure 3. Reduced Morse potential, versus distance, with the ranges parameters, are $c=0.12 \mathrm{~nm}^{-1}$, and the depths $D_{0}=0.15 \mathrm{eV}, D_{0}^{\prime}=5 \times 10^{-3} \mathrm{eV}, U_{0}=5 \times 10^{-2} \mathrm{eV}$. 


$$
\alpha=a \sqrt{\frac{1+\left(\frac{b}{a}\right)^{2} \frac{U_{0}}{D_{0}}+\left(\frac{b}{a}\right)^{2} \frac{D_{0}^{\prime}}{D_{0}}}{1+\frac{1}{D_{0}}\left(U_{0}+D_{0}^{\prime}\right)}}
$$

according to the values of the parameters, we notice that this last parameter is lower than $a$, so the width of the potential decreases. On the other hand, the dissociation energy of the pair $D$ (the depth of potential) increases. Note this potential describes not only the hydrogen bonds and the repulsive interactions of phosphate groups in DNA but all interactions in the system [14]. Let comment on this form of potential: the value $z=0$ corresponds to a closed base pair as in the Ising model, but now $z$ can increase continuously to infinity if the two bases separate completely as in DNA denaturation. The variable $z$ can even take negative values, corresponding to a compression of the bond linking the bases with respect to its equilibrium length. Large negative values will be forbidden by steric hindrance, which is introduced in the model by the potential linking the bases in a pair [15].

Now we determine the free energy. The calculation is similar to the one performed by Krumhansl and Schrieffer [23] for the statistical mechanics of the $\phi^{4}$ field. It yields $\mathcal{Z}_{z}=\exp \left(-N \beta \epsilon_{0}\right)$ where $\epsilon_{0}$ is the lowest eigenvalue of the operator. Therefore, we can then compute the free energy of our model as the sum of the different contribution in $\mathcal{Z}$,

$$
\mathcal{F}=-k_{B} T \ln \mathcal{Z}=\mathcal{F}_{0}-\frac{N k_{B} T}{2} \ln \left(2 \pi m k_{B} T\right)+N \epsilon_{0},
$$

where $\mathcal{F}_{0}=-k_{B} T \ln Z_{0}$, is the free energy of the free membrane, with, $\mathcal{Z}_{0}=q\left(2 \pi k_{B} T\right)^{1 / 2}\left(\kappa q^{4}+\sigma q^{2}\right)^{-1 / 2}$.

The following step is to determine $\epsilon_{0}$. The presence of the membrane and thermal fluctuations affects the eigenfunctions and eigenvalues of DNA molecules, so, $\epsilon_{0}=\epsilon_{0}(h)$ and $\varphi_{0}=\varphi_{0}(r, h)$. [20] The ground state energy $\epsilon_{0}$, as is well-known in quantum mechanics, is alternatively obtained by a variational method, by varying a variational function $\varphi_{0}(r, h(\rho)), \epsilon_{0}$ is given the following relation

$$
\epsilon_{0}=\min \left[\int \mathrm{d}^{3} \boldsymbol{r} \varphi_{0}(\boldsymbol{r}, h) \mathcal{O} \varphi_{0}(\boldsymbol{r}, h)\right]
$$

Since we have interested, by the distance of separation of the base pair of the DNA molecule, one stands in space united dimensional, to facilitate our development further, we use the relation [20]

$$
\varphi(\boldsymbol{r}, h)=\frac{1}{L} \varphi(z-h(\rho))
$$

where $\varphi(z)$ is the ground state variational function of the $1 \mathrm{D}$ free energy operator,

$$
\mathcal{O}(z)=\frac{1}{2 K^{\prime} \beta} \frac{\mathrm{d}^{2}}{\mathrm{~d} z^{2}}-\beta V_{t o t}^{\prime}(z)
$$


where $V_{t o t}^{\prime}(z)=D\left[\mathrm{e}^{-2 \alpha z_{n}}-2 \mathrm{e}^{-\alpha z_{n}}\right]-\left(\varepsilon_{i}-s-D\right)$. With necessary ingredients, the expression of the minimum free energy $\epsilon_{0}$ can be written as follows

$$
\epsilon_{m}=\min \left[\frac{1}{\mathcal{S}} \int \mathrm{d}^{2} \boldsymbol{\rho}\left(\nabla_{\rho} h(\rho)\right)^{2}\langle K\rangle_{\varphi_{0}}+\epsilon_{0}\right],
$$

where the pure DNA-molecule contribution is

$$
\epsilon_{0}=\langle K\rangle_{\varphi_{0}}+\beta\left\langle V_{t o t}^{\prime}(z)\right\rangle_{\varphi_{0}},
$$

With

$$
\begin{gathered}
\langle K\rangle_{\varphi_{0}}=\frac{1}{2 K \beta} \int_{0}^{+\infty} \mathrm{d} z \varphi_{0}(z) \frac{\mathrm{d}^{2} \varphi_{0}(z)}{\mathrm{d} z^{2}}, \\
\beta\langle W(z)\rangle_{\varphi_{0}}=-\frac{1}{2} \int_{0}^{+\infty} \mathrm{d} z \varphi_{0}(z) V \varphi_{0}(z) .
\end{gathered}
$$

Bay a Fourier transformation for $h(\rho)=L^{-1} \sum_{q} h(q) \exp (i \boldsymbol{q} \cdot \boldsymbol{\rho})$ one can reduce the partition function to another form

$$
\begin{aligned}
\mathcal{Z} & =\left(2 \pi m k_{B} T\right)^{N / 2} \times \mathrm{e}^{-N \epsilon_{0}} \mathcal{D} h(\boldsymbol{q}) \exp \left[-\frac{\beta}{2 q}\left(\kappa q^{4}+\tilde{\sigma} q^{2}\right)|h(\boldsymbol{q})|^{2}\right] \\
& =\left(2 \pi m k_{B} T\right)^{N / 2} \times \exp \left(-N \epsilon_{0}\right) q\left(2 \pi k_{B} T\right)^{1 / 2}\left(\kappa q^{4}+\tilde{\sigma} q^{2}\right)^{-1 / 2},
\end{aligned}
$$

Here

$$
\tilde{\sigma}=\sigma+\frac{N}{2 S \beta}\langle K\rangle_{\varphi_{0}},
$$

is the renormalized surface tension at the state $\varphi=\varphi_{0}$ corresponding to the minimum of $\epsilon_{0}(h)$, given by the Equation (20). From the partition function, we obtain the total free energy base per pair induced by its interaction with membrane

$$
\frac{\beta \Delta F}{N}=f_{0}=\min (f)
$$

where

$$
\frac{f}{N}=-k_{B} T \ln \mathcal{Z}+k_{B} T \ln \mathcal{Z}_{0}
$$

With

$$
\mathcal{Z}_{0}=\operatorname{Tr} \mathrm{e}^{-\beta \mathcal{H}_{m}}=\sum_{q}\left(2 \pi k_{B} T\right)^{1 / 2}\left(\kappa q^{4}+\sigma q^{2}\right)^{-1 / 2},
$$

is the partition function of the nude membrane. Thus, we have the following expression of the free energy

$$
\frac{f}{N k_{B} T}=\frac{1}{2 N_{q}} \ln \left[\frac{\left(\kappa q^{4}+\tilde{\sigma} q^{2}\right)}{\kappa q^{4}+\sigma q^{2}}\right]-\frac{1}{2} \ln \left(2 m k_{B} T\right)+\epsilon_{0},
$$

where the first term is the membrane contribution. The condition that $f$ is the minimum for $\varphi=\varphi_{0}$ leads to the new following Schrödinger-like equation 


$$
-\frac{1}{2 K_{\text {Reff }} \beta^{2}} \frac{\mathrm{d}^{2} \varphi_{0}}{\mathrm{~d} z^{2}}+D\left[\mathrm{e}^{-2 \alpha z}-2 \mathrm{e}^{-\alpha z}\right] \varphi_{0}=\left(\epsilon_{0}-s-D\right) \varphi_{0},
$$

this equation for $\varphi_{0}$ minimizing $\epsilon_{0}(h)$, Equation (21), where $K_{R}$ is the renormalized spring constant given by

$$
\frac{1}{K_{R}}=\frac{1}{K^{\prime}}\left(1+\frac{1}{2 S_{q}} \frac{q^{2} k_{B} T}{\kappa q^{4}+\tilde{\sigma} q^{2}}\right),
$$

it is easy to find

$$
K_{R}=K^{\prime}(1+\tau)^{-1}
$$

where $\tau$ is given by the following equation

$$
\tau=\frac{k_{B} T}{16 \pi \kappa} \ln \left[\frac{\kappa(\pi / l)^{4}+\tilde{\sigma}(\pi / l)^{2}}{\kappa(\pi / L)^{4}+\tilde{\sigma}(\pi / L)^{2}}\right]<1,
$$

here $I$ and $L$ are the microscopic and macroscopic lengths that define the upper and lower wave number cutoffs. From the relationship (28a) we conclude that there is a supplementary force added to its previous $f=f_{0}+\Delta f$ such as $\boldsymbol{f}=K_{R}\left(z_{n}-z_{n-1}\right) \boldsymbol{n}, \quad \boldsymbol{f}_{0}=K^{\prime}\left(z_{n}-z_{n-1}\right) \boldsymbol{n}$ and $\Delta \boldsymbol{f}=-\tau K^{\prime}\left(z_{n}-z_{n-1}\right) \boldsymbol{n}, \quad(\boldsymbol{n}$ unit vector), the sign (-) signifies that this force is the inverse of the longitudinal force. These later have two different effects, one pulling the nucleotide, and the other returning it to its balanced position (similar to the nucleotide in each strand a mass attached by two springs). One deducts that the undulation of the membrane, decreases the elasticity constant $K$, so every strand in the double helix becomes more elastic.

\section{Exact Study of Denaturation Transition}

\subsection{Eigenvalue and Ground State}

Equation (27) is formally identical to the Schrödinger equation for a particle in a Morse potential so that it can be solved exactly [25]. So, the melting temperature of DNA adsorbed on a fluctuating membrane is given by

$$
T_{d}=T_{d}^{0}(1+\eta)
$$

where $T_{d}^{0}=2 \sqrt{2 K D_{0}} / a k_{B}$ is the denaturation temperature in the absence of membrane [14], and the quantity $\eta$ is given by the following equation:

$$
\begin{aligned}
\eta= & -\frac{\tau}{2}-\frac{\theta_{e q}^{2}}{8}+\frac{\theta_{e q}^{2}}{16} \tau+\frac{D_{0}^{\prime}+U_{0}}{2 D_{0}}\left(1-\frac{\theta_{e q}^{2}}{8}+\frac{\theta_{e q}^{2}}{16} \tau-\frac{\tau}{2}\right)+\frac{U_{0}}{2 D_{0}}\left(1-\frac{b^{2}}{a^{2}}\right) \\
& +\frac{D_{0}^{\prime}}{2 D_{0}}\left(1-\frac{c^{2}}{a^{2}}\right)+\left(\frac{b U_{0}}{2 a D_{0}}\right)^{2}+\frac{D_{0}^{\prime} U_{0}}{\left(2 a D_{0}\right)^{2}}\left(b^{2}+c^{2}\right)-\left(\frac{c U_{0}}{2 a D_{0}}\right)^{2} .
\end{aligned}
$$

According to the values of the parameters $\eta=0.30871$, it is deduced that the presence of the membrane increases the denaturation temperature of the DNA molecule. Also, this temperature depends on all the parameters of the problem.

Let's comment this result: like a set of external factors, the presence of the cell 
membrane leads to a change in the melting temperature of DNA, the presence of the membrane fluctuations increasing the melting temperature denaturation temperature. According to the parameters, this temperature increases by $\sim 30 \%$. Therefore, when the temperature increasing, the fluctuations of membrane increasing also, which implies that the membrane undergoes a phase transition from a flat phase to a crumpled phase, i.e. its orientations in different points of the membrane are decorrelated, where the persistence length $\xi_{P} \simeq l \exp \left(4 \pi \kappa / 3 k_{\beta} T\right)$, is above the linear size $L$, [26]. A very recent work [27], show a good agreement between experience and numeric study, that the temperature change affects the DNA twist, and suggest that the untwisting of DNA with temperature is predominantly due to changes in DNA structure for defined backbone substates.

The eigenfunction of this system is given by

$$
\varphi_{0}(z)=\tilde{A}_{0}(2 \delta)^{\delta-1 / 2} \mathrm{e}^{-\alpha(\delta-1 / 2) z-\delta \mathrm{e}^{-\alpha z}},
$$

where $\delta=(\beta / \alpha) \sqrt{2 D K_{\text {eff }}}$, and $\tilde{A}_{0}=1 / \sqrt{\Gamma(2 \delta)}$ it is the normalization constant, this equation can be rewritten according to the wave function of the DNA in the absence of the membrane we obtain

$$
\varphi_{0}(z)=\phi_{0}(z) F(z),
$$

where $\phi_{0}(z)=A_{0}(2 \delta)^{\delta_{0}-1 / 2} \mathrm{e}^{-\alpha\left(\delta_{0}-1 / 2\right) z-\delta_{0} \mathrm{e}^{-\alpha z}}$ is the eigenfunction of DNA with $A_{0}=1 / \sqrt{\Gamma\left(2 \delta_{0}\right)}$, and $F(z)$ is the function given by the following relation

$$
\begin{aligned}
F(z)= & \sqrt{1+\eta} \exp \left[\delta_{0} \eta-\left(\delta_{0} \eta-a z \delta_{0} \mu-a z \delta_{0} \eta \mu\right) \mathrm{e}^{-a z}\right] \\
& \times \exp \left[-a z\left(\delta_{0} \mu-\frac{\mu}{2}+\delta_{0} \eta+\delta_{0} \eta \mu\right)\right],
\end{aligned}
$$

with $\delta_{0}=(\beta / a) \sqrt{2 D_{0} K}$, is a constant for a free DNA, and $\mu$, is given by

$$
\begin{aligned}
\mu= & \frac{U_{0}}{2 D_{0}}\left(\frac{b^{2}}{a^{2}}-1\right)+\frac{D_{0}^{\prime}}{2 D_{0}}\left(\frac{c^{2}}{a^{2}}-1\right)-\left(\frac{b U_{0}}{2 a D_{0}}\right)^{2} \\
& -\frac{D_{0}^{\prime} U_{0}}{\left(2 a D_{0}\right)^{2}}\left(b^{2}+c^{2}\right)-\left(\frac{c D_{0}^{\prime}}{2 a D_{0}}\right)^{2} .
\end{aligned}
$$

We notice the eigenfunction is amplified, by a factor $F(z) \sim \sqrt{1+\eta}$. The eigenvalue of the associated ground state

$$
\epsilon_{0}=\epsilon_{0}^{\prime}+\Delta \epsilon_{0}
$$

where $\epsilon_{0}^{\prime}$, it is the eigenvalue of free DNA given by

$$
\epsilon_{0}^{\prime}=\frac{a}{\beta} \sqrt{\frac{2 D_{0}}{K}}-\frac{a^{2}}{2 \beta^{2} K}+\frac{1}{2 \beta} \ln \left(\frac{\beta K}{2 \pi}\right),
$$

and $\Delta \epsilon_{0}$, is the excess energy of ground state its expression is

$$
\Delta \epsilon_{0}=\left(D_{0}-\frac{T_{d}^{0} k_{B}}{2}\right)\left(\frac{T}{T_{d}^{0}}\right)+\left[D_{0}-U_{0}-D_{0}^{\prime}+U_{0}\left(\frac{b}{a}\right)^{2}+D_{0}^{\prime}\left(\frac{c}{a}\right)^{2}\right]\left(\frac{T}{T_{d}^{0}}\right)^{2}
$$

It is noted that the thermal fluctuations of the membrane disturb the eigenva- 
lue of the DNA molecule, as well as the associated wave function. According to the values of the parameters included in Equation (31a), the difference $\Delta \epsilon_{0}$, is very low, so the energy specter of $\epsilon_{0}$, stay near to that $\epsilon_{0}^{\prime}$, we conclude that membrane undulations cause a small perturbation of molecule DNA

\subsection{Thermodynamic Magnitudes at the Critical Point}

\subsubsection{Eigenvalue and Ground State}

We are recalled that to study of the denaturation transition of the DNA molecule, the important quantity which gives a measure of the extent of denaturation of the molecule is the mean stretching of the hydrogen bonds, which given by

$$
\langle z\rangle=\int \varphi_{0}(z)^{2} z \mathrm{~d} z,
$$

We take the results obtained in the case of unbinding transition from two interacting manifolds (strings or bilayer membranes), we find that near the transition temperature (is the similar case) [28], this average distance scale exactly as follows

$$
\langle z\rangle=\frac{1}{\alpha}\left(\frac{T_{d}-T}{T_{d}}\right)^{-1}, \quad T \rightarrow T_{d} .
$$

This average distance is plotted in Figure 4. One notices that the pace of the curve coincides with the one to find by the continuum approximation and the exact numerical calculation [14]. All methods show a divergence of the mean distance at $T=T_{d}$, corresponding to the stretch of links hydrogens, from a given temperature. We concluded that, for the weak values of $\langle z\rangle$, the DNA-molecules are closed and opened for the strong values.

\subsubsection{Surface Tension and Specific Heat}

A quantity that reflects the ability of the molecule of DNA adsorbed on a fluctuating membrane to accumulate energy in thermal form, it is the specific heat. Before calculating the specific heat, we attempt to determine the expression of $\Delta \sigma$. We consider below critical behaviors near $T_{d}$. In terms of small reduced temperature $t=\left(T_{d}-T\right) / T_{d}>0$, we have all ingredients to find the expression of the surface tension depending on the temperature difference

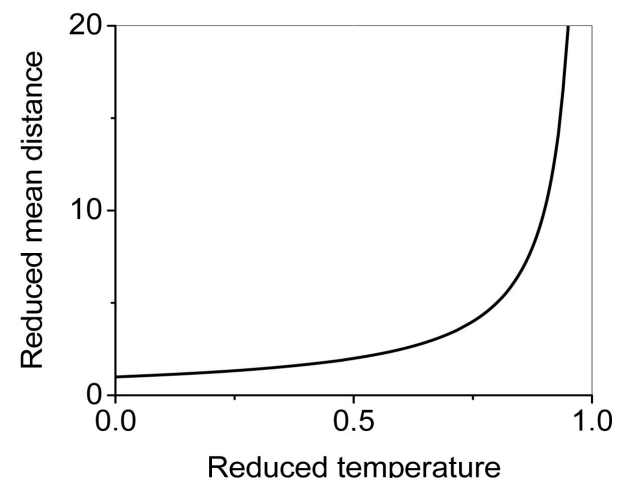

Figure 4. Variation of the reduced mean distance $\langle z\rangle$ versus reduced temperature. 


$$
\tilde{\sigma}=\sigma+\Delta \sigma
$$

where

$$
\Delta \sigma \sim \frac{N \alpha^{2}}{8 S K_{R} \beta^{2} e}\left(\frac{T_{d}-T}{T_{d}}\right)
$$

this expression is according to the difference in the temperature. A simple dimensional analysis shows that $[\Delta \sigma]=j \cdot L^{-2}$. It is necessary to note that $\Delta \sigma=0$ at $T=T_{d}$.

From the relationship (26) we can derive the specific heat

$$
\mathcal{C}_{v}=-T \frac{\partial^{2} \mathcal{F}}{\partial T^{2}}
$$

This specific heat includes a regular part, which is continuous at a transition point, and a singular part, which behaves as a power law with an exponent.

$$
\mathcal{C}_{v}=\mathcal{C}_{v}^{s i}(T)+\mathcal{C}_{v}^{r e g}(T) \text {, }
$$

with regular part given by

$$
\mathcal{C}_{v}^{r e g}(T)=N \frac{T}{T_{d}^{0}}\left[D_{0}-U_{0}-D_{0}^{\prime}+U_{0}\left(\frac{b}{a}\right)^{2}+D_{0}^{\prime}\left(\frac{c}{a}\right)^{2}\right] .
$$

Our objective is to study the critical comportment of the specific heat at denaturation temperature (singular part), therefore we get the following expression

$$
\mathcal{C}_{v} \sim \mathcal{C}_{v}^{s i}(T) \sim\left|\frac{T_{d}^{0}-T}{T_{d}^{0}}\right|^{-\alpha},
$$

with a factor, where $\alpha=1$, is a critical exponent. Our system is immersed in three-dimensional space ( $d=3$ ), but the study of the spherical model has explicitly showed the role of the space dimension on critical phenomena. This model allows calculating exactly the critical properties for an arbitrary space dimension. In this case $\alpha=(4-d) /(d-2)$, (in spherical model $2<d<4$ ), since $d=3$ we have $\alpha=1$, it is a universal critical exponent, the specific heat plotted in Figure 5 .

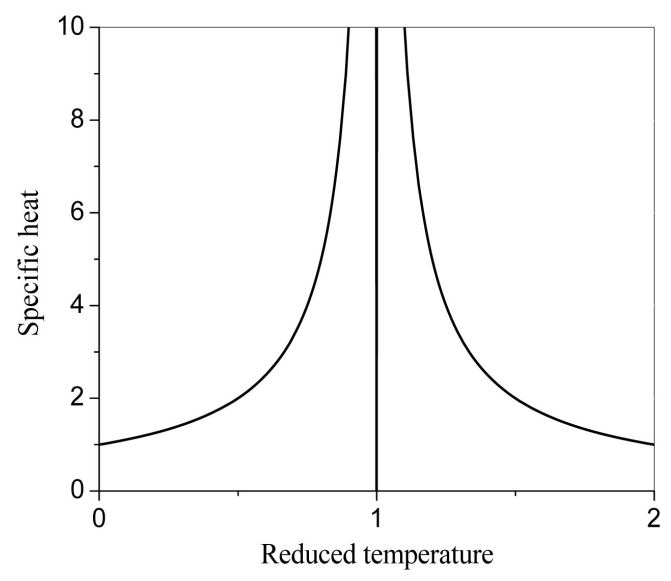

Figure 5. The variation of the specific heat $\mathcal{C}_{v}$, versus reduced temperature. 


\section{Conclusions and Remarks}

In this work, we have studied the influence of the thermal fluctuations membrane on the properties thermodynamics of DNA molecule adsorbed on the fluid membrane. We formulated the problem in terms of a mesoscopic-level partition function and free energy, using the approach variational method, which renormalizes the elasticity constant of DNA. The undulations of the membrane, decrease the elasticity constant $K$. We deduce that nucleotides in each strand vibrate in the longitudinal direction. We can deduce that the modes of vibration also change. This is because the force induced by the membrane, tends to bring the nucleotide back to the equilibrium position.

Consequently, the Schrödinger-like equation which describes the state of the DNA molecule in the absence of the membrane becomes a function of the parameters of our problem. This equation is solved exactly. The resolution of this equation gives the bound state and the associated wave function. From the Schrödinger-like equation, we have determined the melting temperature. This latter depends also on all problem parameters. We have used the eigenfunction associated the ground state to compute the average separation $\langle z\rangle$ between base pairs. This quantity diverges when $T \rightarrow T_{d}$. Another physical quantity has been changed by the adsorption of DNA on the membrane; it is the surface tension $\tilde{\sigma}=\sigma+\Delta \sigma$, where $\Delta \sigma$ vanishes in the vicinity of the denaturation temperature $T_{d}$. In this investigation, we have also found that the heat specific diverges at melting temperature with universal exponent $\alpha=1$.

For the implementation of the denaturation transition of the DNA molecule adsorbed on a membrane, the experimental results concerning the absorption of ultraviolet (UV) by a pure membrane poly (acrylic) acid (PAA) doped with graphite [29], these authors determine the absorbance as a function of the wavelength. They ascended that the absorbance increases with the increase of the concentration of graphite (in the absence of graphite there is a weak absorption [29]). For our case it is necessary to use a pure membrane (absence of graphite and DNA) and UV with a fixed wavelength at $260 \mathrm{~nm}$, and then determine the maximum absorbance for different temperature values, and then use a molecule of DNA adsorbed on a membrane (experiments have been performed on artificial DNAs, which are homopolymers, i.e. have only one type of base pairs [15]), and then measure the absorbance for each temperature value, in the case where the absorbent is maximizing for a certain temperature, that is called the melting or denaturation temperature. It should be noted that as the DNA becomes denatured, its ultraviolet light absorption increases.

The relation (28a) raises interesting questions is the influence of the membrane on the property's DNA, especially the force-extension curve of a single DNA molecule. The first experience obtained in 1992 [30] and repeated with taller precision by several groups a few years ago [31] [32], can be repeated once again for different molecules of DNA with different values of the stiffness constant $\mathrm{K}$, and look at the melting hysteresis comportment. 


\section{Acknowledgements}

We are grateful to Professor M. Benhamou for illuminating discussions.

\section{Conflicts of Interest}

The authors declare no conflicts of interest regarding the publication of this paper.

\section{References}

[1] Watson, J.D. and Crick, F.H.C. (1953) Molecular Structure of Nucleic Acids. Nature, 171, 737-738. https://doi.org/10.1038/171737a0

[2] Saenger, W. (1984) Defining Terms for the Nucleic Acids. In: Principles of Nucleic Acid Structure, Springer, New York, 9-28. https://doi.org/10.1007/978-1-4612-5190-3_2

[3] Asakura, S. and Oosawa, F. (1954) On Interaction between Two Bodies Immersed in a Solution of Macromolecules. The Journal of Chemical Physics, 22, 1255-1256. https://doi.org/10.1063/1.1740347

[4] Zimmerman, S.B. and Minton, A.P. (1993) Macromolecular Crowding: Biochemical, Biophysical, and Physiological Consequences. Annual Review of Biophysics and Biomolecular Structure, 22, 27-65. https://doi.org/10.1146/annurev.bb.22.060193.000331

[5] Westheimer, F.H. (1987) Why Nature Chose Phosphates. Science, 235, 1173-1178. https://doi.org/10.1126/science.2434996

[6] Odijk, T. (2004) Statics and Dynamics of Condensed DNA within Phages and Globules. Philosophical Transactions of the Royal Society of London. Series A: Mathematical, Physical and Engineering Sciences, 362, 1497-1517. https://doi.org/10.1098/rsta.2004.1385

[7] Squires, T.M. and Quake, S.R. (2005) Microfluidics: Fluid Physics at the Nanoliter Scale. Reviews of Modern Physics, 77, 977.

https://doi.org/10.1103/RevModPhys.77.977

[8] Douarche, C., Cortès, R., Roser, S.J., Sikorav, J.L. and Braslau, A. (2008) DNA Adsorption at Liquid/Solid Interfaces. The Journal of Physical Chemistry B, 112, 13676-13679. https://doi.org/10.1021/jp807759d

[9] Buyukdagli, S. and Blossey, R. (2016) Correlation-Induced DNA Adsorption on Like-Charged Membranes. Physical Review E, 94, 042502. https://doi.org/10.1103/PhysRevE.94.042502

[10] Macedo, D.X., Guedes, I. and Albuquerque, E.L. (2014) Thermal Properties of a DNA Denaturation with Solvent Interaction. Physica A: Statistical Mechanics and its Applications, 404, 234-241. https://doi.org/10.1016/j.physa.2014.02.029

[11] Zoli, M. (2011) Thermodynamics of Twisted DNA with Solvent Interaction. The Journal of Chemical Physics, 135, 09B606. https://doi.org/10.1063/1.3631564

[12] Englander, S.W., Kallenbach, N.R., Heeger, A.J., Krumhansl, J.A. and Litwin, S. (1980) Nature of the Open State in Long Polynucleotide Double Helices: Possibility of Soliton Excitations. Proceedings of the National Academy of Sciences of the United States of America, 77, 7222-7226. https://doi.org/10.1073/pnas.77.12.7222

[13] Hwa, T., Marinari, E., Sneppen, K. and Tang, L.-H. (2003) Localization of Denaturation Bubbles in Random DNA Sequences. Proceedings of the National Academy of Sciences the United States of America, 100, 4411-4416. 
https://doi.org/10.1073/pnas.0736291100

[14] Dauxois, T., Peyrard, M. and Bishop, A.R. (1993) Dynamics and Thermodynamics of a Nonlinear Model for DNA Denaturation. Physical Review E, 47, 684. https://doi.org/10.1103/PhysRevE.47.684

[15] Peyrard, M. (2004) Nonlinear Dynamics and Statistical Physics of DNA. Nonlinearity, 17, R1. https://doi.org/10.1088/0951-7715/17/2/R01

[16] Helfrich, W. (1973) Elastic Properties of Lipid Bilayers: Theory and Possible Experiments. Zeitschrift für Naturforschung C, 28, 693-703. https://doi.org/10.1515/znc-1973-11-1209

[17] Ben-Shaul, A. and Gelbart, W.M. (1994) Statistical Thermodynamics of Amphiphile Self-Assembly: Structure and Phase Transitions in Micellar Solutions. In: Micelles, Membranes, Microemulsions, and Monolayers, Springer, New York, 1-104. https://doi.org/10.1007/978-1-4613-8389-5_1

[18] Barbi, M., Lepri, S., Peyrard, M. and Theodorakopoulos, N. (2003) Thermal Denaturation of a Helicoidal DNA Model. Physical Review E, 68, 061909. https://doi.org/10.1103/PhysRevE.68.061909

[19] Lide, D.R. (1995) CRC Handbook of Chemistry and Physics: A Ready-Reference Book of Chemical and Physical Data. CRC Press, Boca Raton.

[20] Sung, W. and Oh, E. (1996) Membrane Fluctuation and Polymer Adsorption. Journal de Physique II, 6, 1195-1206. https://doi.org/10.1051/jp2:1996124

[21] Gradshteyn, I.S. and Ryzhik, I.M. (2014) Table of Integrals, Series, and Products. Academic Press, Cambridge.

[22] Scalapino, D.J., Sears, M. and Ferrell, R.A. (1972) Statistical Mechanics of OneDimensional Ginzburg-Landau Fields. Physical Review B, 6, 3409. https://doi.org/10.1103/PhysRevB.6.3409

[23] Krumhansl, J.A. and Schrieffer, J.R. (1975) Dynamics and Statistical Mechanics of a One-Dimensional Model Hamiltonian for Structural Phase Transitions. Physical Review B, 11, 3535. https://doi.org/10.1103/PhysRevB.11.3535

[24] Bishop, A.R., Krumhansl, J.A. and Trullinger, S.E. (1980) Solitons in Condensed Matter: A Paradigm. Physica D: Nonlinear Phenomena, 1, 1-44. https://doi.org/10.1016/0167-2789(80)90003-2

[25] Morse, P.M. (1929) Diatomic Molecules According to the Wave Mechanics. II. Vibrational Levels. Physical Review, 34, 57. https://doi.org/10.1103/PhysRev.34.57

[26] Nelson, D.R., Piran, T. and Weinberg, S. (Eds.) (2004) Statistical Mechanics of Membranes and Surfaces. 2nd Edition, World Scientific, Singapore. https://doi.org/10.1142/5473

[27] Kriegel, F., Matek, C., Dršata, T., Kulenkampff, K., Tschirpke, S., Zacharias, M. and Lipfert, J. (2018) The Temperature Dependence of the Helical Twist of DNA. Nucleic Acids Research, 46, 7998-8009. https://doi.org/10.1093/nar/gky599

[28] Benhamou, M., El Kinani, R. and Kaidi, H. (2013) Rigorous Study of the Unbinding Transition of Biomembranes and Strings from Morse Potentials. Conference Papers in Science, 2013, Article ID: 320718. https://doi.org/10.1155/2013/320718

[29] Todica, M., Stefan, T., Simon, S., Balasz, I. and Daraban, L. (2014) UV-Vis and XRD Investigation of Graphite-Doped Poly (Acrylic) Acid Membranes. Turkish Journal of Physics, 38, 261-267. https://doi.org/10.3906/fiz-1305-16

[30] Smith, S.B., Finzi, L. and Bustamante, C. (1992) Direct Mechanical Measurements of the Elasticity of Single DNA Molecules by Using Magnetic Beads. Science, 258, 1122-1126. https://doi.org/10.1126/science.1439819 
[31] Smith, S.B., Cui, Y. and Bustamante, C. (1996) Overstretching B-DNA: The Elastic Response of Individual Double-Stranded and Single-Stranded DNA Molecules. Science, 271, 795-799. https://doi.org/10.1126/science.271.5250.795

[32] Cluzel, P., Lebrun, A., Heller, C., Lavery, R., Viovy, J.L., Chatenay, D. and Caron, F. (1996) DNA: An Extensible Molecule. Science, 271, 792-794.

https://doi.org/10.1126/science.271.5250.792 


\section{Appendix}

In this appendix, we will try to develop the expression of following integral

$$
I=\sum_{n=1}^{N} \int_{0}^{\pi / 2} \mathrm{~d} \alpha_{n} \exp ^{\left[-\beta U_{0}\left(1-\exp \left(-b z_{n} \sin \alpha_{n}\right)\right)^{2}\right]} .
$$

To develop this integral we make the first variable change $u_{n}=\exp \left(-a z_{n} \sin \alpha_{n}\right)$, we find

$$
I=-\sum_{n=1}^{N} \int_{1}^{\mathrm{e}^{-a z_{n}}} \mathrm{~d} u_{n} \frac{1}{a z_{n}} \frac{\exp \left(-\beta U_{0}\left(1-u_{n}\right)^{2}\right)}{u_{n} \sqrt{1-\left(\frac{\ln u_{n}}{a z_{n}}\right)^{2}}},
$$

we use also the second variable change as follow $t_{n}=1-u_{n}$, we see that $t_{n} \ll 1$, so the integral becomes [21]

$$
\begin{aligned}
I & \simeq \sum_{n=1}^{N} \frac{1}{a z_{n}} \int_{0}^{1-\mathrm{e}^{-a z_{n}}} \mathrm{~d} t_{n} \exp \left(-\beta U_{0} t_{n}^{2}\right) \\
& \simeq \frac{1}{b z_{n}}\left[\frac{1}{\sqrt{\beta U_{0}}} \mathrm{e}^{-\beta U_{0}\left(1-\mathrm{e}^{-b z_{n}}\right)^{2}}\right] \times \sum_{k=0}^{\infty} \frac{2^{k}\left[\sqrt{\beta U_{0}}\left(1-\mathrm{e}^{-b z_{n}}\right)\right]^{2 k+1}}{(2 k+1) !} .
\end{aligned}
$$

We are restricting to the first order $k=0$, we find a simple development of our integral

$$
I \simeq \exp -\beta U_{0} \sum_{n}\left(1-\mathrm{e}^{-a z_{n}}\right) .
$$

The term $-\beta U_{0} \sum_{n}\left(1-\mathrm{e}^{-a z_{n}}\right)$, is as similar as Morse potential.

\section{Parameters and Nomenclature}

$\theta_{e q}$ : Twist angle between each base pair.

$\alpha_{n}$ : Angle formed between each base pair and membrane.

$n_{\max }$ : The maximal number of monomers adsorbed on the membrane.

$z_{G n}$ : The abscissa of the mass center of point $G_{n}$.

$z_{\text {in }}$ : The abscissa of nucleotide $\left(i=A_{n}, B_{n}\right)$.

$m_{i}$ : Mass of nucleotide $\left(i=A_{n}, B_{n}\right)$.

$m$ : The reduced mass.

$h(x, y)$ : Fluctuation amplitude of the membrane.

$r$. Vector position.

$\rho$ : Projection of $r$ in the reference plane.

$\kappa$ : Membrane bending rigidity.

$\sigma$ : Microscopic membrane surface tension.

$K$ : Harmonic stacking.

$D_{0}$ : Dissociation energy.

a: Inverse length.

$U_{0}$ : Dissociation energy (DNA-membrane).

$b_{0}$ : Inverse length.

$s$. Entropy. 
$\mathcal{Z}:$ Partition function of system.

$\mathcal{F}$ : Free energy.

$\mathcal{F}_{0}$ : Free energy of the free membrane.

$T_{d}$ : Denaturation temperature.

$T_{d}^{0}$ : Denaturation temperature in the absence of membrane.

$\varphi_{0}(z)$ : Eigenfunction.

$\epsilon_{0}$ : Eigenvalue of the associated ground state.

$\langle z\rangle$ : Mean stretching of the hydrogen bonds.

$C_{v}:$ Specific heat. 